\title{
New Definition of the Neutrino Floor for Direct Dark Matter Searches
}

\author{
Ciaran A. J. O'Hare $\odot^{*}$ \\ School of Physics, The University of Sydney, and ARC Centre of Excellence for Dark Matter Particle Physics, \\ New South Wales 2006, Camperdown, Sydney, Australia
}

(Received 8 September 2021; accepted 28 October 2021; published 16 December 2021)

\begin{abstract}
The neutrino floor is a theoretical lower limit on WIMP-like dark matter models that are discoverable in direct detection experiments. It is commonly interpreted as the point at which dark matter signals become hidden underneath a remarkably similar-looking background from neutrinos. However, it has been known for some time that the neutrino floor is not a hard limit, but can be pushed past with sufficient statistics. As a consequence, some have recently advocated for calling it the "neutrino fog" instead. The downside of current methods of deriving the neutrino floor are that they rely on arbitrary choices of experimental exposure and energy threshold. Here we propose to define the neutrino floor as the boundary of the neutrino fog, and develop a calculation free from these assumptions. The technique is based on the derivative of a hypothetical experimental discovery limit as a function of exposure, and leads to a neutrino floor that is only influenced by the systematic uncertainties on the neutrino flux normalizations. Our floor is broadly similar to those found in the literature, but differs by almost an order of magnitude in the sub-GeV range, and above $20 \mathrm{GeV}$.
\end{abstract}

DOI: 10.1103/PhysRevLett.127.251802

Introduction.-Modern experiments searching for dark matter (DM) in the form of weakly interacting massive particles (WIMPs) have become rather large $[1,2]$. It has been anticipated for some time [3-6] that these underground detectors might one day become large enough to detect not just DM, but astrophysical neutrinos as well. In fact, it appears as though the first detection of solar neutrinos in a xenon-based detector is just around the corner [7]. Fittingly, the community has begun to collate a rich catalog of novel physics to be done with our expanding multipurpose network of large underground detectors [8-27].

Unfortunately for WIMP enthusiasts, the impending arrival of neutrinos in DM detectors is somewhat bittersweet-being, as they are, essentially the harbingers of the end of conventional searches. These experiments usually look for signals of DM using nuclear recoils-a channel through which neutrinos also generate events via coherent elastic neutrino-nucleus scattering ( $\mathrm{CE} \nu \mathrm{NS})$ [28-30]. It turns out that the recoil signatures of DM and neutrinos look remarkably alike, with different sources of neutrino each masquerading as DM of varying masses and cross sections [31].

Even an irreducible background like neutrinos may not be so problematic were it not for the-sometimes

Published by the American Physical Society under the terms of the Creative Commons Attribution 4.0 International license. Further distribution of this work must maintain attribution to the author(s) and the published article's title, journal citation, and DOI. Funded by SCOAP . sizable-systematic uncertainties on their fluxes. The cross section below which the potential discovery of a DM signal is prohibited due to this uncertainty is what is usually, but not always, labeled the "neutrino floor" [32]: a limit that has since been the subject of many detailed studies [22,23,33-44]. Since 2013 some form of neutrino floor has been shown underneath all experimental results, often billed as an ultimate sensitivity limit [45]. Methods of circumventing the neutrino floor have been proposed [46-48]. However, only directional detection seems to be a realistic strategy for doing so with comparatively low statistics [49-56].

One potentially misleading aspect of the name neutrino floor is the fact that while it does pose an existential threat to DM searches, the floor itself is not solid. First, the severity of the neutrino background - and hence the height of the floor in terms of cross section - is dependent crucially on neutrino flux uncertainties, which are anticipated to improve over time. Second, the DM and neutrino signals are never perfect matches. Even for DM masses and neutrino fluxes with very closely aligned nuclear recoil spectra-like xenon scattering with ${ }^{8} \mathrm{~B}$ neutrinos and a $6 \mathrm{GeV}$ WIMP - they are not precisely the same. This means that with a large enough number of events, the spectra should be distinguishable [46]. This fact implies that neutrinos present not a floor, but perhaps a "fog:" a region of the parameter space where a clear distinction between signal and background is challenging, but not impossible.

The fogginess of the neutrino floor has become somewhat better appreciated recently $[36,48,57]$. However it is something that is rarely visualized: usually just a single 
neutrino floor limit is plotted. The most common version shown relies on an interpolation of several discovery limits for a set of somewhat arbitrary thresholds and exposures. It is true that given the softness of the neutrino fog, insisting upon a hard boundary will always be slightly arbitrary, however it should be possible to devise a simpler and selfconsistent definition.

Since direct detection experiments will venture into the neutrino fog imminently, it is timely to update and refine our definitions. In this Letter we propose a new definition of the neutrino floor that situates it at the edge of the neutrino fog. We aim for this definition to (i) not depend upon arbitrary choices for experimental thresholds, or absolute numbers of observed neutrino events, (ii) have a single consistent statistical meaning, and (iii) be flexible to future improvements to neutrino flux measurements. The result of this effort can be found in Fig. 1, contrasted against previously used definitions. The technique for calculating this limit is explained graphically in Fig. 2.

Neutrinos versus DM.-To begin, we need to define a DM model to frame our discussion around. We adopt the following DM-nucleus scattering rate,

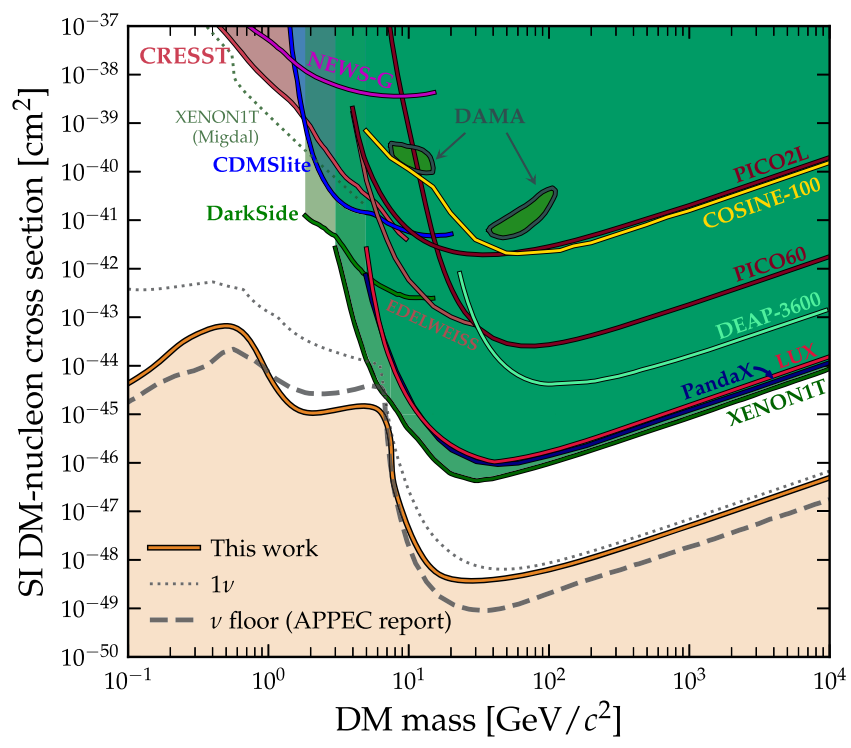

FIG. 1. Present exclusion limits on the spin-independent DMnucleon cross section (assuming equal proton or neutron couplings) [7,58-71]. Beneath these limits we show three definitions of the neutrino floor for a xenon target. The previous discoverylimit-based neutrino floor calculation shown by the dashed line is taken from the recent APPEC report [72] (based on the technique of Ref. [32]). The envelope of 90\% C.L. exclusion limits seeing one expected neutrino event is shown as a dotted line. The result of our work is the solid orange line. We define this notion of the neutrino floor to be the boundary of the neutrino fog, i.e., the cross section at which any experiment sensitive to a given value of $m_{\chi}$ leaves the standard Poissonian regime and begins to be saturated by the background.

$$
\frac{d R_{\chi}}{d E_{r}}=\rho_{0} \frac{\mathcal{C} \sigma}{2 m_{\chi} \mu^{2}} F^{2}\left(E_{r}\right) g\left(v_{\min }\right),
$$

where $\rho_{0}$ is the local DM density, $m_{\chi}$ is the DM mass, $\sigma$ is some DM-nucleon cross section, $\mu$ is the DM-nucleon reduced mass, $\mathcal{C}$ is a nucleus-dependent constant that coherently enhances the rate, and $F\left(E_{r}\right)$ is the form factor that suppresses it at high energies. Finally, $g\left(v_{\min }\right)$ is the mean inverse DM speed above the minimal speed required to produce a recoil with energy $E_{r}$. The latter is found by integrating the lab-frame DM velocity distribution. We assume the time-averaged standard halo model, with parameters summarized in Ref. [73]. Alternative halo models will lead to different neutrino floors, and including the unknown velocity distribution as an additional systematic uncertainty will act to raise the neutrino floor in general [33]. For this initial explanation we will frame the discussion around the spin-independent (SI) isospin-conserving DMnucleon cross section $\sigma \equiv \sigma_{p}^{\mathrm{SI}}$, which is the canonical cross section that experimental collaborations most frequently set exclusion limits on. The scattering rate for this model is enhanced by $\mathcal{C}=A^{2}$, for a target with $A$ nucleons.

Neutrinos can scatter elastically off nuclei and produce recoils with very similar spectra to the ones found by evaluating Eq. (1). Currently, the only measurement of CELNS is by COHERENT [74,75], but it is well understood in the standard model $[28,29]$. Theoretical uncertainties, for example, from the running of the Weinberg

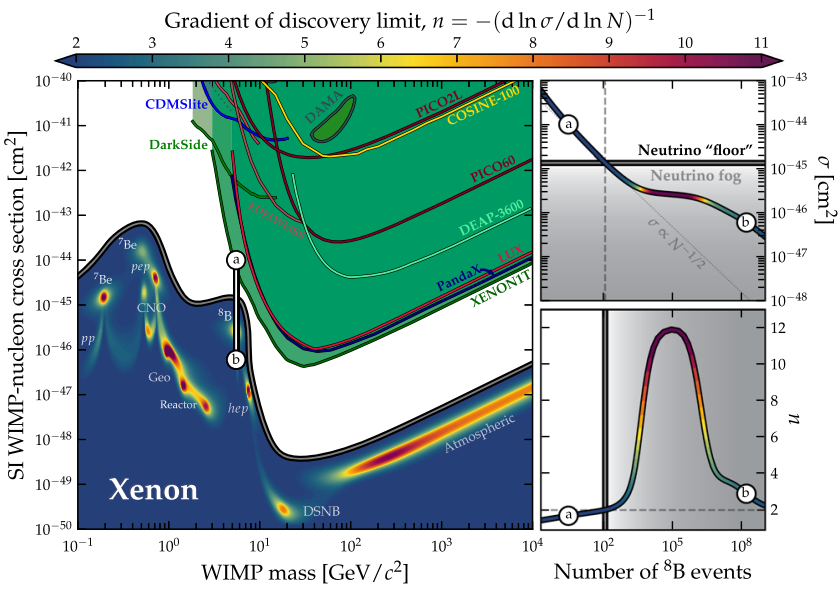

FIG. 2. A graphical description of the technique we adopt to map the neutrino fog and plot its boundary. In the main panel we show the spin-independent DM parameter space, coloring the section below the neutrino floor by the value of $n$, defined as the index with which a discovery limit scales with the number of background events, i.e., $\sigma \propto N^{-1 / n}$. The neutrino fog is defined to be the regime for which $n>2$, with the neutrino floor being the cross section for a given mass where this transition occurs. The top right panel shows the evolution of $\sigma$ with $N$ at $m_{\chi}=5.5 \mathrm{GeV}$ between the two cross sections labeled "a" and "b" on the main panel. 
angle [76], or the nuclear form factor [38], are subdominant to those on the neutrino fluxes [44]. We assume a Weinberg angle of $\sin ^{2} \theta_{W}=0.2387$, and for both neutrino and SI DM scattering we use the standard Helm form factor [77]. Summaries of the calculation of the CE $\nu$ NS cross section $d \sigma_{\nu N}\left(E_{\nu}\right) / \mathrm{d} E_{r}$ can be found in, e.g., Refs. [32,46,54,78].

The recoil energy spectra are then found by integrating the differential CELNS cross section multiplied by the neutrino flux. We adopt the same neutrino flux model as in Ref. [54] (Table I), so we will only briefly summarize some pertinent details. Further information about neutrino fluxes can be found in Ref. [79].

Solar neutrinos generated in nuclear fusion reactions in the Sun form the largest flux at Earth for $E_{\nu} \lesssim 11 \mathrm{MeV}$. These will be the primary source of CE $\nu$ NS events for most DM detectors and will limit discovery around $m_{\chi} \sim 10 \mathrm{GeV}$. The Sun's nuclear energy generation is well understood, and in the case of the most important flux of neutrino from ${ }^{8} \mathrm{~B}$ decay, the corresponding flux normalization, is also measured precisely [80-86]. For the less well-measured components, several theoretical calculations of the solar neutrino fluxes are on the market (see, e.g., Ref. [87] for a recent review). Here we adopt the Barcelona 2016 calculation of the GS98 high-metallicity standard solar model [88]. We adopt the quoted uncertainty on each flux normalization, with the exception of ${ }^{8} \mathrm{~B}$ which we give a $2 \%$ uncertainty in line with global fits of neutrino data [89]. After ${ }^{8} \mathrm{~B}$ neutrinos, the most important solar fluxes are the two neutrino lines from electron capture by ${ }^{7} \mathrm{Be}$, which mimic the signal for sub-GeV masses-these come with $6 \%$ uncertainties.

Geoneutrinos are a constant flux of antineutrinos produced in radioactive decays of mainly uranium, thorium, and potassium in the Earth. For concreteness, we use spectra from Ref. [90] and normalize our geoneutrino fluxes to Gran Sasso, with corresponding uncertainties ranging from $17 \%-25 \%$ [91]. Geoneutrinos impede the discovery at $m_{\chi} \sim \mathrm{GeV}$, but only for cross sections $\sigma_{p}^{\mathrm{SI}} \lesssim 10^{-47} \mathrm{~cm}^{2}$.

Nuclear reactors generate another source of antineutrinos and influence the floor at slightly higher masses. We assume the fission fractions and average energy releases from Ref. [92] combined with the spectra from Ref. [93] before summing over all nearby nuclear reactors to Gran Sasso [94].

The diffuse supernova neutrino background (DSNB) is the cumulative flux of neutrinos from the cosmological history of core-collapse supernovae, and is relevant for the neutrino floor in a small mass window around $20 \mathrm{GeV}$. We adopt the fluxes parameterised in terms of three effective neutrino temperatures for the different flavour contributions, and place a 50\% uncertainty on the all-flavor flux [95].

Atmospheric neutrinos originate from the scattering of high-energy cosmic rays. The low-energy tail of the flux is small $\Phi \sim 10 \mathrm{~cm}^{-2} \mathrm{~s}^{-1}$, but is the dominant background at high recoil energies. We use the theoretical flux model for
$13 \mathrm{MeV}-1 \mathrm{GeV}$ atmospheric neutrinos from FLUKA simulations [96], placing the recommended $20 \%$ theoretical uncertainty. The final exposures of experiments like DARWIN [97] and Argo [98], may reach the high mass section of the neutrino floor set by this background. Since many WIMP-like models with viable cosmologies (see, e.g., Refs. [99-107] for a truncated sample) populate the neutrino fog in this regime, it is a critical part to try to reach.

Statistics.-Our parameters of interest are the DM mass and cross section, as well some nuisance parameters in the form of the neutrino flux normalizations $\boldsymbol{\Phi}=$ $\left\{\Phi^{1}, \ldots, \Phi^{n_{\nu}}\right\}$. We use a binned likelihood written as the product of the Poisson probability in each bin, multiplied by Gaussian likelihood functions for the uncertainties on each neutrino flux normalization:

$\mathcal{L}(\sigma, \boldsymbol{\Phi})=\prod_{i=1}^{N_{\text {bins }}} \mathcal{P}\left[N_{\text {obs }}^{i} \mid N_{\chi}^{i}+\sum_{j=1}^{n_{\nu}} N_{\nu}^{i}\left(\Phi^{j}\right)\right] \prod_{j=1}^{n_{\nu}} \mathcal{G}\left(\Phi^{j}\right)$.

The Gaussian distributions have standard deviations given by the systematic uncertainties that we just discussed. The Poisson probabilities at the $i$ th bin are taken for an observed number of events $N_{\mathrm{obs}}^{i}$, given an expected number of signal events $N_{\chi}^{i}$ and the sum of the expected number of neutrino events for each flux $N_{\nu}^{i}\left(\Phi^{j}\right)$. We bin events logarithmically between $10^{-4}$ and $150 \mathrm{keV}$. The former threshold is clearly not in any way realistic, however the main advantage of our definition of the neutrino floor will be that it is not based on absolute numbers of events. Our choice of threshold is simply to allow us to map the neutrino floor down to $m_{\chi}=0.1 \mathrm{GeV}$, but crucially it does not impact the height of the limit at other masses.

If we have two models, a background-only model $\mathcal{M}_{\sigma=0}$, and a signal + background model $\mathcal{M}$, we can test for $\sigma>0$ using the following statistic,

$$
q_{0}= \begin{cases}-2 \ln \left[\frac{\mathcal{L}\left(0, \hat{\hat{\mathbf{\phi}}} \mid \mathcal{M}_{\sigma=0}\right)}{\mathcal{L}(\hat{\sigma}, \hat{\mathbf{\Phi}} \mid \mathcal{M})}\right] & \hat{\sigma}>0 \\ 0 & \hat{\sigma} \leq 0\end{cases}
$$

where $\mathcal{L}$ is maximized at $\hat{\hat{\boldsymbol{\Phi}}}$ when $\sigma$ is set to 0 , and $(\hat{\sigma}, \hat{\mathbf{\Phi}})$ when $\sigma$ is a free parameter. The model $\mathcal{M}_{\sigma=0}$ is a special case of $\mathcal{M}$, obtained by fixing one parameter to the boundary of its allowed space. Therefore Chernoff's theorem [108] holds, and $q_{0}$ should be asymptotically distributed according to $\frac{1}{2} \chi_{1}^{2}+\frac{1}{2} \delta(0)$ when $\mathcal{M}$ is true [109].

Evaluating $q_{0}$ while avoiding the need to collect many Monte Carlo realizations for every point in the parameter space, we exploit the Asimov dataset [110]. This is a hypothetical scenario in which the observation exactly matches the expectation for a given model, i.e., $N_{\mathrm{obs}}^{i}=$ $N_{\text {exp }}^{i}$ for all $i$. The test statistic computed assuming this dataset asymptotes towards the median of the chosen model's $q_{0}$ distribution [110]. In high-statistics analyses 
such as ours, this turns out to be an extremely good approximation, and has been demonstrated multiple times in similar calculations $[33,54,57]$. We fix $N_{\text {obs }}$ to be the expected number of neutrino and DM events, and require a threshold test statistic of $q_{0}>9$. Therefore our limits are defined as the expected $3 \sigma$ discovery limits.

The neutrino fog.-We can now explain how the neutrino background impacts the discovery of DM. The critical factor to understand is the systematic uncertainty on the background. The way to think about this is to imagine a very feeble DM signal that closely matches one of the background components. Such a signal is saturated not just when the number of signal events is simply less than the background, but when that excess of events is smaller than the statistical fluctuations of the background. The regime of parameter space where this occurs is what we define as the neutrino fog.

We can quantify the neutrino fog by considering how some discovery limit $\sigma$ decreases as the exposure or the number of observed background events $N$, increases. The limit evolves through three distinct scalings. Initially, when the experiment is essentially background free, $N<1$, the limit evolves as $\sigma \propto N^{-1}$. Then, as $N$ increases, the limit transitions into Poissonian background subtraction: $\propto 1 / \sqrt{N}$. Eventually, as the number of events increases further, any would-be detectable DM signal disappears beneath the scale of potential background fluctuations, and the limit is stalled at $\sigma \propto \sqrt{\left(1+N \delta \Phi^{2}\right) / N}$ [32].

If the DM signal and $\mathrm{CE} \nu \mathrm{NS}$ background were identical, the saturation regime would persist for arbitrarily large $N$. However there is rarely a value of $m_{\chi}$ for which the background perfectly matches the signal. In theory one could always collect enough statistics to distinguish the two via their tails or some other broad spectral features. So eventually the limit will emerge from saturation, and the $\sigma \propto N^{-1 / 2}$ scaling returns, but only by the time $N$ has grown very large. In fact, reaching beyond the saturation regime across the mass range studied here would consume the entire world's supply of atmospheric xenon.

The "opacity" of the neutrino fog can therefore be visualized by plotting some gradient of this discovery limit. Let us define the index $n=-(d \ln \sigma / d \ln N)^{-1}$, so that $n=2$ under normal Poissonian subtraction, and $n>2$ when there is saturation (see also Refs. [111-114] for analyses in other contexts that introduce quantities similar to this). The value of this index for each point in the neutrino fog is shown by the color scale in Fig. 2. Seeing as for every value of $m_{\chi}$ there is a value of $\sigma$ where $n$ crosses 2 , we can join these points together in a contour to form the boundary of the neutrino fog, or neutrino floor.

The result of this procedure is the solid line in Fig. 1. There, we compared our result alongside two previous definitions quoted frequently in the literature. The dashed line shows the computation which follows the technique described in Refs. [32,46], with this specific limit taken from Ref. [72]. The technique invoked there involves the interpolation of two limits: a low-threshold or low-exposure one that captures solar neutrinos, and a high-threshold or high-exposure one that excludes solar neutrinos and captures atmospheric and supernovae neutrinos. The two discovery limits are for 400 observed neutrino events so as to put them somewhere around the systematics dominated part of the fog for certain masses.

We also showed a "one-neutrino" contour, which is formed from the envelope of a series of background-free 90\% C.L. exclusion limits (2.3 events) with increasing thresholds that have exposures large enough to see one expected neutrino event. These limits have a similar shape but are higher in cross section. One-neutrino contours have an advantage in that they are easy to calculate, but come with the downside that they do not encode any information about the DM or neutrino spectral degeneracy, and do not incorporate systematic uncertainties.

We compare our new neutrino floors for six different targets in Fig. S1 of the Supplemental Material [115]. In the SI space, they are largely similar, with the general trend that the kinematics of scattering off lighter target nuclei means their floors are pushed to higher masses. Helium is the extreme case, because Eq. (1) enters the asymptotic $R_{\chi} \propto \rho_{0} / m_{\chi}$ scaling for much lighter masses than other targets, hence why the neutrino floor above $10 \mathrm{GeV}$ is significantly higher, and is set by solar neutrinos rather than atmospheric neutrinos. The broad features observed in these figures largely persist for other interactions, such as spin-dependent scattering. We show examples of these in Fig. S2 [115].

Finally, in the right-hand panel of Fig. 3 we show how our definition of the neutrino floor is adaptable to improvements in the flux estimates. The dashed line imagines that we have obtained a factor of 10 improvement in the atmospheric neutrino uncertainty, i.e., down to $\sim 2 \%$, whereas the dot-dashed line imagines that all solar flux estimates are improved by a factor of 10 . These scenarios are intended to be illustrative, rather than in anticipation of any specific improvements. Nevertheless, with a flock of large-scale neutrino observatories on the horizon [116-120], it is not unreasonable to expect some improvement to our knowledge of the neutrino fluxes [54,121-125].

Discussion. - Given the imminent arrival of the neutrino background in underground WIMP-like DM searches, we have decided to revisit and refine the neutrino floor, or perhaps more appropriately, neutrino fog. Our floor can be interpreted as the boundary of the neutrino fog in a statistically meaningful way. It marks the point at which any experiment will start to be limited by the background: a cross section that is influenced only by overlap between the DM and neutrino spectra, and the background's systematic uncertainties.

In contrast to prior calculations, our definition of the neutrino floor is not based on arbitrary absolute numbers of 

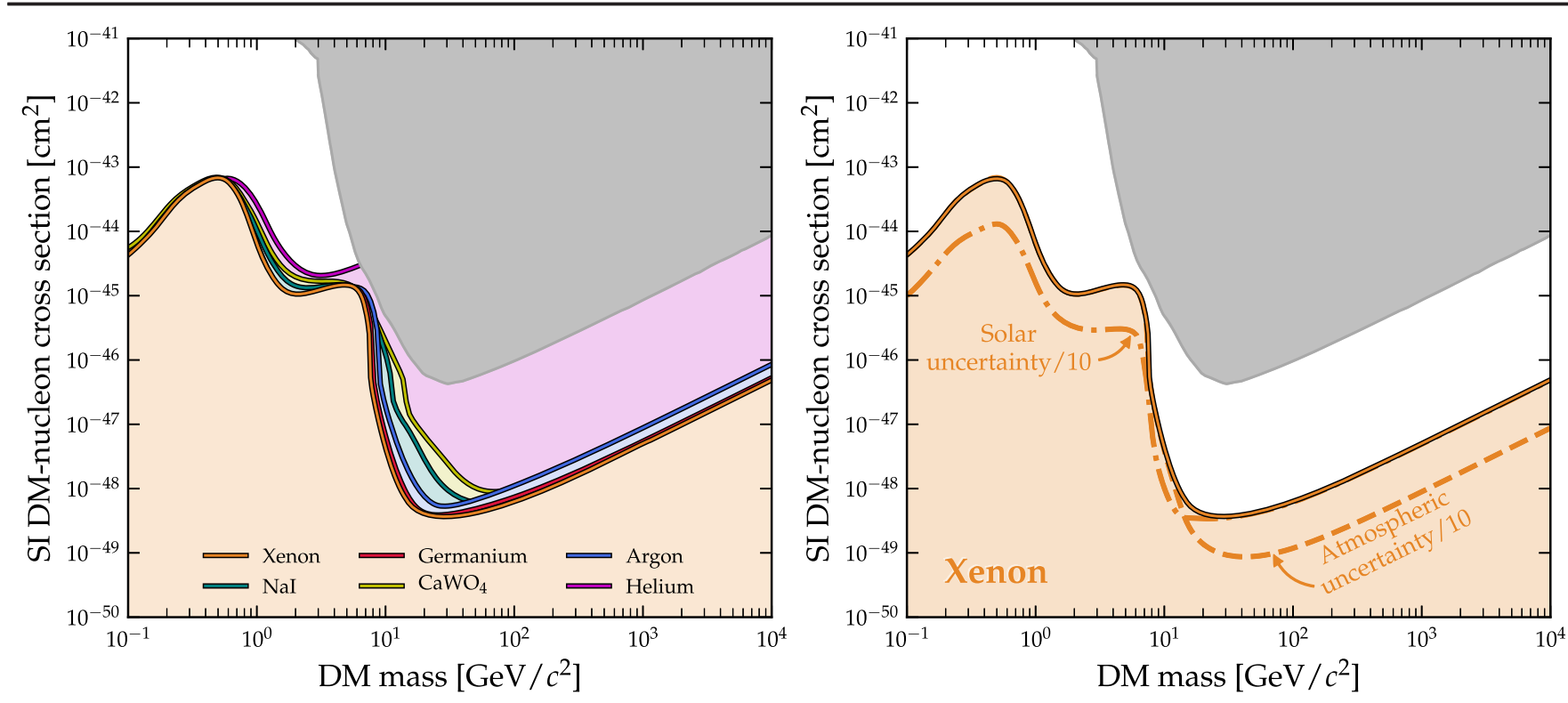

FIG. 3. Left: The neutrino floor for various popular direct detection targets. The general trend is for lighter targets to have floors shifted towards higher masses. The full topology of the neutrino fog for these same targets is shown in Fig. S1 of the Supplemental Material [115]. Right: xenon's neutrino floor, showing the effect of improvements to neutrino flux uncertainties. The solid line shows our baseline calculation, whereas the dot-dashed and dashed lines show the result after reducing the systematic uncertainties on the solar and atmospheric fluxes by a factor of 10 .

events, but on the derivative of the discovery limit. As such we arrive at a limit that does not depend upon the recoil energy threshold, so long as one does not attempt to calculate the limit for masses that only scatter below the chosen threshold. This also means that we do not need to interpolate multiple limits together to map the floor across a wider mass range, and can do so with a single calculation.

The main quantitative differences between our result and those found in the literature are the following. First, we can see, for instance, in Fig. 1, that our neutrino floor is noticeably higher in the sub-GeV region where ${ }^{7} \mathrm{Be}$ neutrinos mimic the signal, as well as the high mass region where the same is true of atmospheric neutrinos. Previous calculations that used fixed exposures, ended up placing the neutrino floor deeper into the systematics dominated regime for those masses compared to others. On the other hand, the shoulder in the neutrino floor around $6 \mathrm{GeV}$ is lower in our case. This is because we have adopted a systematic uncertainty on the ${ }^{8} \mathrm{~B}$ flux of $2 \%$ which is based on a global fit of neutrino data [89]. The previous calculation of the neutrino floor assumed a $15 \%$ systematic uncertainty coming from the solar model estimation.

We have applied our new technique to map the neutrino fog for a range of targets (Fig. S1 [115]) as well as a few different DM interactions (Fig. S2 [115]). In the right-hand panel of Fig. 3 we also showed how our neutrino floor can be updated in the future as neutrino observatories make continued refinements to measured fluxes. Our technique is such that these improvements can be incorporated without changing the definition of the neutrino floor.

Data available at [126].

This work was supported by The University of Sydney.

*ciaran.ohare@sydney.edu.au

[1] M. Battaglieri et al., U.S. cosmic visions: New ideas in dark matter 2017, Community Report, arXiv:1707.04591, http://lss.fnal.gov/archive/2017/conf/fermilab-conf-17-282ae-ppd-t.pdf.

[2] M. Schumann, Direct detection of WIMP dark matter: Concepts and status, J. Phys. G 46, 103003 (2019).

[3] J. Monroe and P. Fisher, Neutrino backgrounds to dark matter searches, Phys. Rev. D 76, 033007 (2007).

[4] J.D. Vergados and H. Ejiri, Can solar neutrinos be a serious background in direct dark matter searches?, Nucl. Phys. B804, 144 (2008).

[5] L. E. Strigari, Neutrino coherent scattering rates at direct dark matter detectors, New J. Phys. 11, 105011 (2009).

[6] A. Gutlein, C. Ciemniak, F. von Feilitzsch, N. Haag, M. Hofmann, C. Isaila, T. Lachenmaier, J.-C. Lanfranchi, L. Oberauer, and S. Pfister, Solar and atmospheric neutrinos: Background sources for the direct dark matter search, Astropart. Phys. 34, 90 (2010).

[7] E. Aprile et al. (XENON Collaboration), Search for Coherent Elastic Scattering of Solar ${ }^{8} \mathrm{~B}$ Neutrinos in the XENON1T Dark Matter Experiment, Phys. Rev. Lett. 126, 091301 (2021). 
[8] R. Harnik, J. Kopp, and P. A. N. Machado, Exploring $\nu$ signals in dark matter detectors, J. Cosmol. Astropart. Phys. 07 (2012) 026.

[9] M. Pospelov, Neutrino physics with dark matter experiments and the signature of new baryonic neutral currents, Phys. Rev. D 84, 085008 (2011).

[10] J. Billard, L. E. Strigari, and E. Figueroa-Feliciano, Solar neutrino physics with low-threshold dark matter detectors, Phys. Rev. D 91, 095023 (2015).

[11] D. Franco et al., Solar neutrino detection in a large volume double-phase liquid argon experiment, J. Cosmol. Astropart. Phys. 08 (2016) 017.

[12] M. Schumann, L. Baudis, L. Butikofer, A. Kish, and M. Selvi, Dark matter sensitivity of multi-ton liquid xenon detectors, J. Cosmol. Astropart. Phys. 10 (2015) 016.

[13] L. E. Strigari, Neutrino floor at ultralow threshold, Phys. Rev. D 93, 103534 (2016).

[14] J. B. Dent, B. Dutta, S. Liao, J. L. Newstead, L. E. Strigari, and J.W. Walker, Probing light mediators at ultralow threshold energies with coherent elastic neutrino-nucleus scattering, Phys. Rev. D 96, 095007 (2017).

[15] J.-W. Chen, H.-C. Chi, C. P. Liu, and C.-P. Wu, Lowenergy electronic recoil in xenon detectors by solar neutrinos, Phys. Lett. B 774, 656 (2017).

[16] D. G. Cerdeño, M. Fairbairn, T. Jubb, P. A. N. Machado, A. C. Vincent, and C. Bœhm, Physics from solar neutrinos in dark matter direct detection experiments, J. High Eenrgy Phys. 05 (2016) 118; Erratum, J. High Eenrgy Phys. 09 (2016) 048.

[17] B. Dutta and L.E. Strigari, Neutrino physics with dark matter detectors, Annu. Rev. Nucl. Part. Sci. 69, 137 (2019).

[18] R. F. Lang, C. McCabe, S. Reichard, M. Selvi, and I. Tamborra, Supernova neutrino physics with xenon dark matter detectors: A timely perspective, Phys. Rev. D 94, 103009 (2016).

[19] E. Bertuzzo, F. F. Deppisch, S. Kulkarni, Y. F. Perez Gonzalez, and R. Zukanovich Funchal, Dark matter and exotic neutrino interactions in direct detection searches, J. High Energy Phys. 04 (2017) 073.

[20] B. Dutta, S. Liao, L. E. Strigari, and J. W. Walker, Nonstandard interactions of solar neutrinos in dark matter experiments, Phys. Lett. B 773, 242 (2017).

[21] M. Leyton, S. Dye, and J. Monroe, Exploring the hidden interior of the Earth with directional neutrino measurements, Nat. Commun. 8, 15989 (2017).

[22] D. Aristizabal Sierra, N. Rojas, and M. Tytgat, Neutrino non-standard interactions and dark matter searches with multi-ton scale detectors, J. High Energy Phys. 03 (2018) 197.

[23] C. Bœhm, D. G. Cerdeño, P. A. N. Machado, A. OlivaresDel Campo, and E. Reid, How high is the neutrino floor?, J. Cosmol. Astropart. Phys. 01 (2019) 043.

[24] N. F. Bell, J. B. Dent, J. L. Newstead, S. Sabharwal, and T. J. Weiler, Migdal effect and photon bremsstrahlung in effective field theories of dark matter direct detection and coherent elastic neutrino-nucleus scattering, Phys. Rev. D 101, 015012 (2020).

[25] J. L. Newstead, L. E. Strigari, and R. F. Lang, Detecting CNO solar neutrinos in next-generation xenon dark matter experiments, Phys. Rev. D 99, 043006 (2019).
[26] J. Aalbers et al. (DARWIN Collaboration), Solar neutrino detection sensitivity in DARWIN via electron scattering, Eur. Phys. J. C 80, 1133 (2020).

[27] D. S. Akerib et al. (LZ Collaboration), Projected sensitivities of the LUX-ZEPLIN (LZ) experiment to new physics via low-energy electron recoils, arXiv:2102.11740.

[28] D. Z. Freedman, Coherent neutrino nucleus scattering as a probe of the weak neutral current, Phys. Rev. D 9, 1389 (1974).

[29] D. Z. Freedman, D. N. Schramm, and D. L. Tubbs, The weak neutral current and its effects in stellar collapse, Annu. Rev. Nucl. Sci. 27, 167 (1977).

[30] A. Drukier and L. Stodolsky, Principles and applications of a neutral current detector for neutrino physics and astronomy, Phys. Rev. D 30, 2295 (1984).

[31] J. Billard, F. Mayet, G. Bosson, O. Bourrion, O. Guillaudin et al., In situ measurement of the electron drift velocity for upcoming directional dark matter detectors, J. Instrum. 9, P01013 (2014).

[32] J. Billard, L. Strigari, and E. Figueroa-Feliciano, Implication of neutrino backgrounds on the reach of next generation dark matter direct detection experiments, Phys. Rev. D 89, 023524 (2014).

[33] C. A. J. O'Hare, Dark matter astrophysical uncertainties and the neutrino floor, Phys. Rev. D 94, 063527 (2016).

[34] J. B. Dent, B. Dutta, J. L. Newstead, and L. E. Strigari, Effective field theory treatment of the neutrino background in direct dark matter detection experiments, Phys. Rev. D 93, 075018 (2016).

[35] J. B. Dent, B. Dutta, J. L. Newstead, and L. E. Strigari, Dark matter, light mediators, and the neutrino floor, Phys. Rev. D 95, 051701(R) (2017).

[36] G. B. Gelmini, V. Takhistov, and S. J. Witte, Casting a wide signal net with future direct dark matter detection experiments, J. Cosmol. Astropart. Phys. 07 (2018) 009.

[37] M. C. Gonzalez-Garcia, M. Maltoni, Y. F. Perez-Gonzalez, and R. Zukanovich Funchal, Neutrino discovery limit of dark matter direct detection experiments in the presence of non-standard interactions, J. High Energy Phys. 07 (2018) 019.

[38] D. K. Papoulias, R. Sahu, T. S. Kosmas, V. K. B. Kota, and B. Nayak, Novel neutrino-floor and dark matter searches with deformed shell model calculations, Adv. High Energy Phys. 2018, 1 (2018).

[39] R. Essig, M. Sholapurkar, and T.-T. Yu, Solar neutrinos as a signal and background in direct-detection experiments searching for sub-GeV dark matter with electron recoils, Phys. Rev. D 97, 095029 (2018).

[40] J. Wyenberg and I. M. Shoemaker, Mapping the neutrino floor for direct detection experiments based on dark matter-electron scattering, Phys. Rev. D 97, 115026 (2018).

[41] M. Nikolic, S. Kulkarni, and J. Pradler, The neutrino-floor in the presence of dark radation, arXiv:2008.13557.

[42] V. Munoz, V. Takhistov, S. J. Witte, and G. M. Fuller, Exploring the origin of supermassive black holes with coherent neutrino scattering, arXiv:2102.00885.

[43] R. Calabrese, D. F. G. Fiorillo, G. Miele, S. Morisi, and A. Palazzo, Primordial black hole dark matter evaporating on the neutrino floor, arXiv:2106.02492. 
[44] D. A. Sierra, V. De Romeri, L. J. Flores, and D. K. Papoulias, Impact of COHERENT measurements, cross section uncertainties and new interactions on the neutrino floor, arXiv:2109.03247.

[45] E. Gibney, Last chance for WIMPs: Physicists launch allout hunt for dark-matter candidate, Nature (London) 586, 344 (2020).

[46] F. Ruppin, J. Billard, E. Figueroa-Feliciano, and L. Strigari, Complementarity of dark matter detectors in light of the neutrino background, Phys. Rev. D 90, 083510 (2014).

[47] J.H. Davis, Dark matter vs. neutrinos: The effect of astrophysical uncertainties and timing information on the neutrino floor, J. Cosmol. Astropart. Phys. 03 (2015) 012.

[48] S. Sassi, A. Dinmohammadi, M. Heikinheimo, N. Mirabolfathi, K. Nordlund, H. Safari, and K. Tuominen, Solar neutrinos and dark matter detection with diurnal modulation, Phys. Rev. D 104, 063037 (2021).

[49] C. A. J. O'Hare, A. M. Green, J. Billard, E. FigueroaFeliciano, and L. E. Strigari, Readout strategies for directional dark matter detection beyond the neutrino background, Phys. Rev. D 92, 063518 (2015).

[50] P. Grothaus, M. Fairbairn, and J. Monroe, Directional dark matter detection beyond the neutrino bound, Phys. Rev. D 90, 055018 (2014).

[51] F. Mayet et al., A review of the discovery reach of directional dark matter detection, Phys. Rep. 627, 1 (2016).

[52] C. A. J. O'Hare, B. J. Kavanagh, and A. M. Green, Timeintegrated directional detection of dark matter, Phys. Rev. D 96, 083011 (2017).

[53] T. Franarin and M. Fairbairn, Reducing the solar neutrino background in dark matter searches using polarized helium-3, Phys. Rev. D 94, 053004 (2016).

[54] C. A. J. O'Hare, Can we overcome the neutrino floor at high masses?, Phys. Rev. D 102, 063024 (2020).

[55] S. Vahsen et al., CYGNUS: Feasibility of a nuclear recoil observatory with directional sensitivity to dark matter and neutrinos, arXiv:2008.12587.

[56] S. E. Vahsen, C. A. J. O'Hare, and D. Loomba, Directional recoil detection, Annu. Rev. Nucl. Part. Sci. 71, 189 (2021).

[57] A. Gaspert, P. Giampa, and D. E. Morrissey, Neutrino backgrounds in future liquid noble element dark matter direct detection experiments, arXiv:2108.03248.

[58] R. Agnese et al. (SuperCDMS Collaboration), Search for low-mass dark matter with CDMSlite using a profile likelihood fit, Phys. Rev. D 99, 062001 (2019).

[59] G. Adhikari et al., An experiment to search for dark-matter interactions using sodium iodide detectors, Nature (London) 564, 83 (2018).

[60] A. H. Abdelhameed et al. (CRESST Collaboration), First results from the CRESST-III low-mass dark matter program, Phys. Rev. D 100, 102002 (2019).

[61] R. Bernabei et al., First model independent results from DAMA/LIBRA-phase2, Universe 4, 116 (2018).

[62] C. Savage, G. Gelmini, P. Gondolo, and K. Freese, Compatibility of DAMA/LIBRA dark matter detection with other searches, J. Cosmol. Astropart. Phys. 04 (2009) 010 .
[63] P. Agnes et al. (DarkSide Collaboration), Low-Mass Dark Matter Search with the DarkSide-50 Experiment, Phys. Rev. Lett. 121, 081307 (2018).

[64] R. Ajaj et al. (DEAP Collaboration), Search for dark matter with a 231-day exposure of liquid argon using DEAP-3600 at SNOLAB, Phys. Rev. D 100, 022004 (2019).

[65] L. Hehn et al. (EDELWEISS Collaboration), Improved EDELWEISS-III sensitivity for low-mass WIMPs using a profile likelihood approach, Eur. Phys. J. C 76, 548 (2016).

[66] D. S. Akerib et al. (LUX Collaboration), Results from a Search for Dark Matter in the Complete LUX Exposure, Phys. Rev. Lett. 118, 021303 (2017).

[67] Q. Arnaud et al. (NEWS-G Collaboration), First results from the NEWS-G direct dark matter search experiment at the LSM, Astropart. Phys. 97, 54 (2018).

[68] X. Cui et al. (PandaX-II Collaboration), Dark Matter Results From 54-Ton-Day Exposure of PandaX-II Experiment, Phys. Rev. Lett. 119, 181302 (2017).

[69] C. Amole et al. (PICO Collaboration), Improved dark matter search results from PICO-2L Run 2, Phys. Rev. D 93, 061101 (2016).

[70] C. Amole et al. (PICO Collaboration), Dark Matter Search Results from the PICO-60 $\mathrm{C}_{3} \mathrm{~F}_{8}$ Bubble Chamber, Phys. Rev. Lett. 118, 251301 (2017).

[71] E. Aprile et al. (XENON Collaboration), Search for Light Dark Matter Interactions Enhanced by the Migdal Effect or Bremsstrahlung in XENON1T, Phys. Rev. Lett. 123, 241803 (2019).

[72] J. Billard et al., Direct detection of dark matter, APPEC Committee Report, arXiv:2104.07634.

[73] N. W. Evans, C. A. J. O'Hare, and C. McCabe, Refinement of the standard halo model for dark matter searches in light of the Gaia Sausage, Phys. Rev. D 99, 023012 (2019).

[74] D. Akimov et al. (COHERENT Collaboration), Observation of coherent elastic neutrino-nucleus scattering, Science 357, 1123 (2017).

[75] D. Akimov et al. (COHERENT Collaboration), First Measurement of Coherent Elastic Neutrino-Nucleus Scattering on Argon, Phys. Rev. Lett. 126, 012002 (2021).

[76] J. Erler and M. J. Ramsey-Musolf, The weak mixing angle at low energies, Phys. Rev. D 72, 073003 (2005).

[77] J. D. Lewin and P. F. Smith, Review of mathematics, numerical factors, and corrections for dark matter experiments based on elastic nuclear recoil, Astropart. Phys. 6, 87 (1996).

[78] K. Scholberg, Prospects for measuring coherent neutrinonucleus elastic scattering at a stopped-pion neutrino source, Phys. Rev. D 73, 033005 (2006).

[79] E. Vitagliano, I. Tamborra, and G. Raffelt, Grand unified neutrino spectrum at Earth: Sources and spectral components, Rev. Mod. Phys. 92, 045006 (2020).

[80] S. Fukuda et al. (Super-Kamiokande Collaboration), Solar ${ }^{8} B$ and hep Neutrino Measurements from 1258 Days of Super-Kamiokande Data, Phys. Rev. Lett. 86, 5651 (2001).

[81] G. Bellini et al. (Borexino Collaboration), Measurement of the solar ${ }^{8} \mathrm{~B}$ neutrino rate with a liquid scintillator target and $3 \mathrm{MeV}$ energy threshold in the Borexino detector, Phys. Rev. D 82, 033006 (2010). 
[82] M. Agostini et al. (Borexino Collaboration), Improved measurement of ${ }^{8} \mathrm{~B}$ solar neutrinos with $1.5 \mathrm{kt} \Delta \mathrm{y}$ of Borexino exposure, Phys. Rev. D 101, 062001 (2020).

[83] K. Abe et al. (Super-Kamiokande Collaboration), Solar neutrino results in Super-Kamiokande-III, Phys. Rev. D 83, 052010 (2011).

[84] S. Abe et al. (KamLAND Collaboration), Measurement of the ${ }^{8} \mathrm{~B}$ Solar Neutrino Flux with the KamLAND liquid scintillator detector, Phys. Rev. C 84, 035804 (2011).

[85] B. Aharmim et al. (SNO Collaboration), Combined analysis of all three phases of solar neutrino data from the Sudbury Neutrino Observatory, Phys. Rev. C 88, 025501 (2013).

[86] M. Anderson et al. (SNO+Collaboration), Measurement of the ${ }^{8} \mathrm{~B}$ solar neutrino flux in $\mathrm{SNO}+$ with very low backgrounds, Phys. Rev. D 99, 012012 (2019).

[87] G. D. O. Gann, K. Zuber, D. Bemmerer, and A. Serenelli, The future of solar neutrinos, Annu. Rev. Nucl. Part. Sci. 71, 491 (2021).

[88] N. Vinyoles, A. M. Serenelli, F. L. Villante, S. Basu, J. Bergström, M. C. Gonzalez-Garcia, M. Maltoni, C. Peña-Garay, and N. Song, A new generation of standard solar models, Astrophys. J. 835, 202 (2017).

[89] J. Bergstrom, M. C. Gonzalez-Garcia, M. Maltoni, C. Pena-Garay, A. M. Serenelli, and N. Song, Updated determination of the solar neutrino fluxes from solar neutrino data, J. High Energy Phys. 03 (2016) 132.

[90] L. Ludhova and S. Zavatarelli, Studying the Earth with geoneutrinos, Adv. High Energy Phys. 2013, 1 (2013).

[91] Y. Huang, V. Chubakov, F. Mantovani, R. L. Rudnick, and W. F. McDonough, A reference earth model for the heatproducing elements and associated geoneutrino flux, Geochem. Geophys. Geosyst. 14, 2003 (2013).

[92] X. B. Ma, W. L. Zhong, L. Z. Wang, Y.X. Chen, and J. Cao, Improved calculation of the energy release in neutron-induced fission, Phys. Rev. C 88, 014605 (2013).

[93] T. A. Mueller et al., Improved predictions of reactor antineutrino spectra, Phys. Rev. C 83, 054615 (2011).

[94] M. Baldoncini, I. Callegari, G. Fiorentini, F. Mantovani, B. Ricci, V. Strati, and G. Xhixha, Reference worldwide model for antineutrinos from reactors, Phys. Rev. D 91, 065002 (2015).

[95] J. F. Beacom, The diffuse supernova neutrino background, Annu. Rev. Nucl. Part. Sci. 60, 439 (2010).

[96] G. Battistoni, A. Ferrari, T. Montaruli, and P. R. Sala, The atmospheric neutrino flux below 100-MeV: The FLUKA results, Astropart. Phys. 23, 526 (2005).

[97] J. Aalbers et al. (DARWIN Collaboration), DARWIN: Towards the ultimate dark matter detector, J. Cosmol. Astropart. Phys. 11 (2016) 017.

[98] S. Sanfilippo et al. (DarkSide Collaboration), DarkSide status and prospects, Nuovo Cimento Soc. Ital. Fis. 42, 79 (2019).

[99] P. Athron et al. (GAMBIT Collaboration), Global fits of GUT-scale SUSY models with GAMBIT, Eur. Phys. J. C 77, 824 (2017).

[100] P. Athron et al. (GAMBIT Collaboration), GAMBIT: The global and modular beyond-the-standard-model inference tool, Eur. Phys. J. C 77, 784 (2017); 78, 98(A) (2018).
[101] C. Beskidt, W. de Boer, D. Kazakov, and S. Wayand, Perspectives of direct detection of supersymmetric dark matter in the NMSSM, Phys. Lett. B 771, 611 (2017).

[102] L. Roszkowski, E. M. Sessolo, and A. J. Williams, Prospects for dark matter searches in the pMSSM, J. High Energy Phys. 02 (2015) 014.

[103] J. Hisano, K. Ishiwata, N. Nagata, and T. Takesako, Direct detection of electroweak-interacting dark matter, J. High Energy Phys. 07 (2011) 005.

[104] A. Kobakhidze and M. Talia, Supersymmetric naturalness beyond MSSM, J. High Energy Phys. 08 (2019) 105.

[105] G. Arcadi, M. Lindner, F. S. Queiroz, W. Rodejohann, and S. Vogl, Pseudoscalar mediators: A WIMP model at the neutrino floor, J. Cosmol. Astropart. Phys. 03 (2018) 042.

[106] M. J. Baker, J. Kopp, and A. J. Long, Filtered Dark Matter at a First Order Phase Transition, Phys. Rev. Lett. 125, 151102 (2020).

[107] C. Arina, A. Beniwal, C. Degrande, J. Heisig, and A. Scaffidi, Global fit of pseudo-Nambu-Goldstone dark matter, J. High Energy Phys. 04 (2020) 015.

[108] H. Chernoff, On the distribution of the likelihood ratio, Ann. Math. Stat. 25, 573 (1954).

[109] S. Algeri, J. Aalbers, K. Dundas Morå, and J. Conrad, Searching for new phenomena with profile likelihood ratio tests, Nat. Rev. Phys. 2, 245 (2020).

[110] G. Cowan, K. Cranmer, E. Gross, and O. Vitells, Asymptotic formulae for likelihood-based tests of new physics, Eur. Phys. J. C 71, 1554 (2011); Erratum, Eur. Phys. J. C 73, 2501 (2013).

[111] T.D. P. Edwards and C. Weniger, swordfish: Efficient forecasting of new physics searches without Monte Carlo, arXiv: 1712.05401.

[112] T. D. P. Edwards, B. J. Kavanagh, and C. Weniger, Assessing Near-Future Direct Dark Matter Searches with Benchmark-Free Forecasting, Phys. Rev. Lett. 121, 181101 (2018).

[113] T. D. P. Edwards, B. J. Kavanagh, C. Weniger, S. Baum, A. K. Drukier, K. Freese, M. Górski, and P. Stengel, Digging for dark matter: Spectral analysis and discovery potential of paleo-detectors, Phys. Rev. D 99, 043541 (2019).

[114] S. Baum, T. D. P. Edwards, K. Freese, and P. Stengel, New projections for dark matter searches with Paleo-detectors, Instruments 5, 21 (2021).

[115] See Supplemental Material at http://link.aps.org/ supplemental/10.1103/PhysRevLett.127.251802 for additional figures showing the full neutrino floors for six alternative targets, as well as two different DM interaction cross sections.

[116] M. Askins et al. (Theia Collaboration), THEIA: An advanced optical neutrino detector, Eur. Phys. J. C 80, 416 (2020).

[117] E. Caden (SNO+ Collaboratoin), Status of the SNO+ experiment, in Proceedings of the 15th International Conference on Topics in Astroparticle and Underground Physics (TAUP 2017) Sudbury, Ontario, Canada, 2017 (IOP Publishing, Bristol, 2017) [J. Phys.: Conf. Ser. 1342 011001 (2020)], 10.1088/1742-6596/1342/1/011001. 
[118] J. F. Beacom et al. (Jinping Collaboration), Physics prospects of the Jinping neutrino experiment, Chin. Phys. C 41, 023002 (2017).

[119] B. Abi et al. (DUNE Collaboration), The DUNE Far Detector Interim Design Report Volume 1: Physics, technology and strategies, arXiv:1807.10334.

[120] F. An et al. (JUNO Collaboration), Neutrino physics with JUNO, J. Phys. G 43, 030401 (2016).

[121] A. G. Cocco, A. Ereditato, G. Fiorillo, G. Mangano, and V. Pettorino, Supernova relic neutrinos in liquid argon detectors, J. Cosmol. Astropart. Phys. 12 (2004) 002 .

[122] Z. Li (Super-Kamiokande, Hyper-Kamiokande Collaborations), Atmospheric neutrinos and proton decay in
Super-Kamiokande and Hyper-Kamiokande, Nucl. Part. Phys. Proc. 287-288, 147 (2017).

[123] F. Capozzi, S. W. Li, G. Zhu, and J. F. Beacom, DUNE as the Next-Generation Solar Neutrino Experiment, Phys. Rev. Lett. 123, 131803 (2019).

[124] G. Zhu, S. W. Li, and J. F. Beacom, Developing the MeV potential of DUNE: Detailed considerations of muoninduced spallation and other backgrounds, Phys. Rev. C 99, 055810 (2019).

[125] K. J. Kelly, P. A. Machado, I. Martinez Soler, S. J. Parke, and Y.F. Perez Gonzalez, Sub-GeV Atmospheric Neutrinos and CP-Violation in DUNE, Phys. Rev. Lett. 123, 081801 (2019).

[126] https://github.com/cajohare/NeutrinoFog. 